УДК $\mathbf{5 1 7 . 9 8}$

\title{
REVERSIBLE AJW-ALGEBRAS
}

\author{
Sh. A. Ayupov, F. N. Arzikulov
}

\begin{abstract}
The main result states that every special $A J W$-algebra can be decomposed into the direct sum of totally irreversible and reversible subalgebras. In turn, every reversible special $A J W$-algebra decomposes into a direct sum of two subalgebras, one of which has purely real enveloping real von Neumann algebra, and the second one contains an ideal, whose complexification is a $\mathrm{C}^{*}$-algebra and the annihilator of this complexification in the enveloping $C^{*}$-algebra of this subalgebra is equal to zero.
\end{abstract}

Mathematics Subject Classification (2000): 17C65, 46L57.

AJW-algebra, reversible AJW-algebra, AW*-algebra, Enveloping $\mathrm{C}^{*}$-algebra.

\section{Introduction}

This article is devoted to abstract Jordan operator algebras, which are analogues of abstract $\mathrm{W}^{*}$-algebras (AW*-algebras) of Kaplansky. These Jordan operator algebras can be characterized as a JB-algebra satisfying the following conditions

(1) in the partially ordered set of all projections any subset of pairwise orthogonal projections has the least upper bound in this JB-algebra;

(2) every maximal associative subalgebra of this JB-algebra is generated by it's projections (i.e. coincides with the least closed subalgebra containing all projections of the given subalgebra).

In the articles $[3,4]$ the second author introduced analogues of annihilators for Jordan algebras and gave algebraic conditions equivalent to (1) and (2). Currently, these JB-algebras are called AJW-algebras or Baer JB-algebras in the literature. Further, in [5] a classification of these algebras has been obtained. It should be noted that many of facts of the theory of JBW-algebras and their proofs hold for AJW-algebras. For example, similar to a JBW-algebra an AJW-algebra is the direct sum of special and purely exceptional Jordan algebras [5].

It is known from the theory of JBW-algebras that every special JBW-algebra can be decomposed into the direct sum of totally irreversible and reversible subalgebras. In turn, every reversible special JBW-algebra decomposes into a direct sum of a subalgebra, which is the hermitian part of a von Neumann algebra and a subalgebra, enveloping real von Neumann algebra of which is purely real $[6,7]$. In this paper we prove a similar result for AJW-algebras, the proof of which requires a different approach. Namely, we prove that for every special AJWalgebra $A$ there exist central projections $e, f, g \in A, e+f+g=1$ such that (1) $e A$ is reversible and there exists a norm-closed two sided ideal $I$ of $C^{*}(e A)$ such that $e A={ }^{\perp}\left({ }^{\perp}\left(I_{s a}\right)_{+}\right)_{+} ;(2) f A$ is reversible and $R^{*}(f A) \cap i R^{*}(f A)=\{0\} ;(3) g A$ is a totally nonreversible AJW-algebra.

(c) 2016 Ayupov Sh. A., Arzikulov F. N. 


\section{Preliminary Notes}

We fix the following terminology and notations.

Let $\mathscr{A}$ be a real Banach $*$-algebra. $\mathscr{A}$ is called a real $\mathrm{C}^{*}$-algebra, if $\mathscr{A}_{c}=\mathscr{A}+i \mathscr{A}=$ $\{a+i b: a, b \in \mathscr{A}\}$, can be normed to become a (complex) $\mathrm{C}^{*}$-algebra, and keeps the original norm on $\mathscr{A}[11]$.

Let $A$ be a JB-algebra, $P(A)$ be a set of all projections of $A$. Further we will use the following standard notations: $\{a b a\}=U_{a} b:=2 a(a b)-a^{2} b,\{a b c\}=a(b c)+(a c) b-(a b) c$ and $\{a A b\}=\{\{a c b\}: c \in A\}$, where $a, b, c \in A$. A JB-algebra $A$ is called an AJW-algebra, if the following conditions hold:

(1) in the partially ordered set $P(A)$ of projections any subset of pairwise orthogonal projections has the least upper bound in $A$;

(2) every maximal associative subalgebra $A_{o}$ of the algebra $A$ is generated by it's projections (i. e. coincides with the least closed subalgebra containing $A_{o} \cap P(A)$ ).

Let

$$
\begin{aligned}
(S)^{\perp}= & \left\{a \in A:(\forall x \in S) U_{a} x=0\right\}, \\
{ }^{\perp}(S)= & \left\{x \in A:(\forall a \in S) U_{a} x=0\right\}, \\
& { }^{\perp}(S)_{+}={ }^{\perp}(S) \cap A_{+} .
\end{aligned}
$$

Then for a JB-algebra $A$ the following conditions are equivalent:

(1) $A$ is an AJW-algebra;

(2) for every subset $S \subset A_{+}$there exists a projection $e \in A$ such that $(S)^{\perp}=U_{e}(A)$;

(3) for every subset $S \subset A$ there exists a projection $e \in A$ such that ${ }^{\perp}(S)_{+}=U_{e}\left(A_{+}\right)$[3].

Let $A$ be a real or complex *-algebra, and let $S$ be a nonempty subset of $A$. Then the set $R(S)=\{x \in A: s x=0$ for all $s \in S\}$ is called the right annihilator of $S$ and the set $L(S)=\{x \in A: x s=0$ for all $s \in S\}$ is called the left annihilator of $S$. A $*$-algebra $A$ is called a Baer *-algebra, if the right annihilator of any nonempty set $S \subseteq A$ is generated by a projection, i.e. $R(S)=g A$ for some projection $g \in A\left(g^{2}=g=g^{*}\right)$. If $S=\{a\}$ then the projection $1-g$ such that $R(S)=g A$ is called the right projection and denoted by $r(a)$. Similarly one can define the left projection $l(a)$. A (real) $\mathrm{C}^{*}$-algebra $A$, which is a Baer (real) *-algebra, is called an (real) $\mathrm{AW}^{*}$-algebra $[1,8]$. Real $\mathrm{AW}^{*}$-algebras were introduced and investigated in $[1,2]$. In these papers it was shown that for a real $\mathrm{AW}^{*}$-algebra $\mathscr{A}$ the $\mathrm{C}^{*}$-algebra $M=\mathscr{A}+i \mathscr{A}$ is not necessarily a complex $\mathrm{AW}^{*}$-algebra.

Let $A$ be an AJW-algebra. By [5, Theorem 2.3] we have the equality $A=A_{I} \oplus A_{I I} \oplus A_{I I I}$, where $A_{I}$ is an A.JW-algebra of type I, $A_{I I}$ is an AJW-algebra of type II and $A_{I I I}$ is an AJWalgebra of type III [5]. By [5, Theorem 3.7] $A_{I}$, in its turn, is a direct sum of the following form

$$
A_{I}=A_{\infty} \oplus A_{1} \oplus A_{2} \oplus \ldots,
$$

where $A_{n}$ for every $n$ either is $\{0\}$ or an AJW-algebra of type $\mathrm{I}_{n}, A_{\infty}$ is a direct sum of AJWalgebras of type $\mathrm{I}_{\alpha}$ with $\alpha$ infinite. If $A=A_{1} \oplus A_{2} \oplus \ldots$ then $A$ is called an AJW-algebra of type $\mathrm{I}_{\text {fin }}$ and denoted by $A_{I_{f i n}}$ and if $A=A_{\infty}$ then $A$ is called an A.JW-algebra of type $\mathrm{I}_{\infty}$ and denoted by $A_{I_{\infty}}$. We say that $A$ is properly infinite if $A$ has no nonzero central modular projection. The fact that an AJW-algebra $A_{I I}$ of type II is a JC-algebra can be proved similar to JBW-algebras [9]. Therefore, it is isomorphic to some AJW-algebra defined in [14] (i. e. to some AJW-algebra of self-adjoint operators), and by virtue of [14] $A_{I I}=A_{I I_{1}} \oplus A_{I I_{\infty}}$, where $A_{I I_{1}}$ is a modular AJW-algebra of type II and $A_{I I_{\infty}}$ is an AJW-algebra of type II, which is properly infinite. So, we have the decomposition

$$
A=A_{I_{f i n}} \oplus A_{I_{\infty}} \oplus A_{I I_{1}} \oplus A_{I I_{\infty}} \oplus A_{I I I}
$$


It is easy to verify that the part $A_{I_{f i n}} \oplus A_{I I_{1}}$ is modular, and $A_{I_{\infty}} \oplus A_{I I_{\infty}} \oplus A_{I I I}$ is properly infinite (i.e. properly nonmodular).

\section{Reversibility of AJW-algebras}

Let $A$ be a special AJW-algebra on a complex Hilbert space $H$. By $R^{*}(A)$ we denote the uniformly closed real *-algebra in $B(H)$, generated by $A$, and by $C^{*}(A)$ the $\mathrm{C}^{*}$-algebra, generated by $A$. Thus the set of elements of kind

$$
\sum_{i=1}^{n} \prod_{j=1}^{m_{i}} a_{i j} \quad\left(a_{i j} \in A\right)
$$

is uniformly dense in $R^{*}(A)$. Let $i R^{*}(A)$ be the set of elements of kind $i a, a \in R^{*}(A)$. Then $C^{*}(A)=R^{*}(A)+i R^{*}(A)[7,13]$.

Lemma 3.1. The set $R^{*}(A) \cap i R^{*}(A)$ is a uniformly closed two sided ideal in $C^{*}(A)$.

$\triangleleft$ If $a, b \in R^{*}(A)$ and $c=i d \in R^{*}(A) \cap i R^{*}(A)$, then $(a+i b) c=a c+i b i d=a c-b d \in R^{*}(A)$. Similarly $(a+i b) c \in i R^{*}(A)$, i. e. $(a+i b) c \in R^{*}(A) \cap i R^{*}(A)$. Since $R^{*}(A) \cap i R^{*}(A)$ is uniformly closed and the set of elements of kind $a+i b, a, b \in A$ is uniformly dense in $C^{*}(A)$, we have $R^{*}(A) \cap i R^{*}(A)$ is a left ideal in $C^{*}(A)$. By the symmetry $R^{*}(A) \cap i R^{*}(A)$ is a right ideal. $\triangleright$

Let $R$ be a $*$-algebra, $R_{s a}$ be the set of all self-adjoint elements of $R$, i. e. $R_{s a}=\{a \in R$ : $\left.a^{*}=a\right\}$.

Definition 3.2. A JC-algebra $A$ is said to be reversible if $a_{1} a_{2} \ldots a_{n}+a_{n} a_{n-1} \ldots a_{1} \in A$ for all $a_{1}, a_{2}, \ldots, a_{n} \in A$.

Similar to JW-algebras we have the following criterion.

Lemma 3.3. An $A J W$-algebra $A$ is reversible if and only if $A=R^{*}(A)_{s a}$.

$\triangleleft \mathrm{It}$ is clear that, if $A=R^{*}(A)_{s a}$, then $A$ is reversible since

$$
\left(\prod_{i=1}^{n} a_{i}+\prod_{i=n}^{1} a_{i}\right)^{*}=\prod_{i=n}^{1} a_{i}+\prod_{i=1}^{n} a_{i} \in R^{*}(A)_{s a}=A,
$$

for all $a_{1}, a_{2}, \ldots, a_{n} \in A$. Conversely, let $A$ be a reversible AJW-algebra. The inclusion $A \subset$ $R^{*}(A)_{s a}$ is evident. If $a=\sum_{i=1}^{n} \prod_{j=1}^{m_{i}} a_{i j} \in R^{*}(A)_{s a}$, then

$$
a=\frac{1}{2}\left(a+a^{*}\right)=\frac{1}{2} \sum_{i=1}^{n}\left(\prod_{j=1}^{m_{i}} a_{i j}+\prod_{j=m_{i}}^{1} a_{i j}\right) \in A .
$$

Hence the converse inclusion holds, i. e. $R^{*}(A)_{s a}=A$. $\triangleright$

Lemma 3.4. Let $A$ be an $A J W$-algebra and let $I$ be a norm-closed ideal of $A$. Then there exists a central projection $g$ such that ${ }^{\perp}\left({ }^{\perp}\left(I_{s a}\right)_{+}\right)_{+}=g A_{+}$.

$\triangleleft$ Since $A$ is an AJW-algebra there exists a projection $g$ in $A$ such that

$$
{ }^{\perp}\left(I_{s a}\right)_{+}=U_{(1-g)}\left(A_{+}\right), \quad{ }^{\perp}\left({ }^{\perp}\left(I_{s a}\right)_{+}\right)_{+}=U_{g}\left(A_{+}\right),
$$

where ${ }^{\perp}(S)_{+}=\left\{x \in A_{+}:(\forall a \in S) U_{a} x=0\right\}$ for $S \subseteq A$.

Let $\left(u_{\lambda}\right)$ be an approximate identity of the JB-subalgebra $I$ and $a$ be an arbitrary positive element in $I$. Then there exists a maximal associative subalgebra $A_{o}$ of $A$ containing $a$. Let 
$v_{\mu}$ be an approximate identity of $A_{o}$. Then $\left(v_{\mu}\right) \subseteq\left(u_{\lambda}\right)$ and $\left\|a v_{\mu}-a\right\| \rightarrow 0$. Let $b \in A_{+}$ and $U_{v_{\mu}} b=0$ for every $\mu$. Then $U_{a} U_{v_{\mu}} b=U_{a v_{\mu}} b=0$ and $U_{c} U_{a v_{\mu}} b=0$, where $c$ is an element in $A$ such that $b=c^{2}$. Hence $U_{c} U_{a v_{\mu}} c^{2}=0,\left(U_{c}\left(a v_{\mu}\right)\right)^{2}=0, U_{c}\left(a v_{\mu}\right)=0$ and $U_{c} U_{c}\left(a v_{\mu}\right)=U_{b}\left(a v_{\mu}\right)=0$ for every $\mu$. We have

$$
\left.\left\|U_{b}\left(a v_{\mu}\right)-U_{b} a\right\|=\| U_{b}\left(a v_{\mu}\right)-a\right) \| \rightarrow 0
$$

because $\left\|a v_{\mu}-a\right\| \rightarrow 0$ and the operator $U_{b}$ is norm-continuous. Hence $U_{b} a=0$. We may assume that $a=d^{2}$ for some element $d \in A$. Then

$$
U_{d} U_{b} a=U_{d} U_{b} d^{2}=\left(U_{d} b\right)^{2}=0, \quad U_{d} b=0 .
$$

Thus $U_{d} U_{d} b=U_{d^{2}} b=U_{a} b=0$. Therefore, if $b \in^{\perp}\left(\left(u_{\lambda}\right)\right)_{+}$then $b \in^{\perp}\left(I_{s a}\right)_{+}$. Hence ${ }^{\perp}\left(\left(u_{\lambda}\right)\right)_{+} \subseteq^{\perp}\left(I_{s a}\right)_{+}$. It is clear that ${ }^{\perp}\left(I_{s a}\right)_{+} \subseteq^{\perp}\left(\left(u_{\lambda}\right)\right)_{+}$and

$$
{ }^{\perp}\left(I_{s a}\right)_{+}={ }^{\perp}\left(\left(u_{\lambda}\right)\right)_{+} \cdot
$$

This implies that ${ }^{\perp}\left(\left(u_{\lambda}\right)\right)_{+}=U_{(1-g)}\left(A_{+}\right)$and

$$
\sup _{\lambda} u_{\lambda}=g
$$

Let us prove that $U_{g}(A)$ is an ideal of $A$. Indeed, let $x$ be an arbitrary element in $A$. Then $U_{x} u_{\lambda} \in I_{s a}$, i.e. $U_{x} u_{\lambda} \in U_{g}(A)$. By [9, Proposition 3.3.6] and the proof of [9, Lemma 4.1.5] we have $U_{x}$ is a normal operator in $A$. Hence

$$
\sup _{\lambda} U_{x} u_{\lambda}=U_{x}\left(\sup _{\lambda} u_{\lambda}\right)=U_{x} g
$$

At the same time

$$
\sup _{\lambda} U_{x} u_{\lambda} \in U_{g}(A)
$$

Hence $U_{x} g \in U_{g}(A)$. By [9, 2.8.10] we have

$$
4(x g)^{2}=2 g U_{x} g+U_{x} g^{2}+U_{g} x^{2}=2 g U_{x} g+U_{x} g+U_{g} x^{2} .
$$

Therefore $(x g)^{2} \in U_{g}(A)$ and $x g \in U_{g}(A)$.

Now, let $y$ be an arbitrary element in $U_{g} A$. Then $y=U_{g} y$ and

$$
x y=\left(U_{g} x+\{g x(1-g)\}+U_{1-g} x\right) U_{g} y=U_{g} x U_{g} y+\{g x(1-g)\} U_{g} y \in U_{g} A
$$

since $\{g x(1-g)\} \in U_{g} A$. Hence $U_{g} A$ is a norm-closed ideal of $A$. Therefore $\{g A(1-g)\}=\{0\}$ and

$$
A=U_{g} A \oplus U_{1-g} A .
$$

This implies that $g$ is a central projection in $A$ and ${ }^{\perp}\left({ }^{\perp}\left(I_{s a}\right)_{+}\right)_{+}=g A_{+} \cdot \triangleright$

Lemma 3.5. Let $A$ be a reversible $A J W$-algebra on a Hilbert space $H$. Then there exist two central projections $e, f$ in $A$ and a norm-closed two sided ideal $I$ of $C^{*}(A)$ such that $e+f=1, e A={ }^{\perp}\left({ }^{\perp}\left(I_{s a}\right)_{+}\right)_{+}$and $R^{*}(f A) \cap i R^{*}(f A)=\{0\}$.

$\triangleleft$ Let $I=R^{*}(A) \cap i R^{*}(A)$. Since $A$ is reversible by Proposition 3.3 we have $I_{s a} \subseteq A$. By $[7,3.1] I$ is a two sided ideal of $C^{*}(A)$. Hence $I_{s a}$ is an ideal of the AJW-algebra $A$. By Proposition 3.4 we have there exists a central projection $g$ such that ${ }^{\perp}\left({ }^{\perp}\left(I_{s a}\right)_{+}\right)_{+}=g A_{+}$. It is clear that $g$ is a central projection also in $C^{*}(A)$.

By the definitions of $I$ and $g$ we have

$$
R^{*}((1-g) A) \cap i R^{*}((1-g) A)=\{0\} . \triangleright
$$


Lemma 3.6. Let $A$ be an $A J W$-algebra and let $J$ be the set of elements $a \in A$ such that $b a c+c^{*} a b^{*} \in A$ for all $b, c \in C^{*}(A)$. Then $J$ is a norm-closed ideal in $A$. Moreover $J$ is a reversible $A J W$-algebra.

$\triangleleft$ Let $a, b \in J, s, t \in C^{*}(A)$. Then

$$
s(a+b) t+t^{*}(a+b) s^{*}=\left(s a t+t^{*} a s^{*}\right)+\left(s b t+t^{*} b s^{*}\right) \in A,
$$

i. e. $J$ is a linear subspace of $A$. Now, if $a \in J, b \in A, s, t \in C^{*}(A)$, then

$$
s(a b+b a) t+t^{*}(a b+b a) s^{*}=\left(s a(b t)+(b t)^{*} a s^{*}\right)+\left((s b) a t+t^{*} a(s b)^{*}\right) \in A,
$$

i. e. $J$ is a norm-closed ideal of $A$.

Let $a_{1} \in J, a_{2}, \ldots, a_{n} \in A$ and $a=\prod_{i=2}^{n} a_{i}$. Then $a_{1} a+a^{*} a_{1} \in A$ by the definition of $J$. Let us show that $a_{1} a+a^{*} a_{1} \in J$; then, in particular, in the case of $a_{2}, \ldots, a_{n} \in J$ this will imply that $J$ is reversible. For all $b, c \in C^{*}(A)$ we have

$$
b\left(a_{1} a+a^{*} a_{1}\right) c+c^{*}\left(a_{1} a+a^{*} a_{1}\right) b^{*}=\left(b a_{1}(a c)+(a c)^{*} a_{1} b^{*}\right)+\left(\left(b a^{*}\right) a_{1} c+c^{*} a_{1}\left(b a^{*}\right)^{*}\right) \in A,
$$

i. e. $a_{1} a+a^{*} a_{1} \in J . \triangleright$

Definition 3.7.An AJW-algebra $A$ is said to be totally nonreversible, if the ideal $J$ in Lemma 3.6 is equal to $\{0\}$, i. e. $J=\{0\}$.

Theorem 3.8. Let $A$ be a special $A J W$-algebra. Then there exist central projections $e, f, g \in A, e+f+g=1$ such that

(1) $J=(e+f) A, J$ is the ideal from Lemma 3.6;

(2) $e A$ is reversible and there exists a norm-closed two sided ideal $I$ of $C^{*}(e A)$ such that $e A={ }^{\perp}\left({ }^{\perp}\left(I_{s a}\right)_{+}\right)_{+}$;

(3) $f A$ is reversible and $R^{*}(f A) \cap i R^{*}(f A)=\{0\}$;

(4) $g A$ is a totally nonreversible $A J W$-algebra and

$$
g A=\sum_{\omega \in \Omega} C\left(Q_{\omega}, \mathbf{R} \oplus H_{\omega}\right),
$$

where $\Omega$ is a set of indices, $\left\{Q_{\omega}\right\}_{\omega \in \Omega}$ is an appropriate family of extremal compacts and $\left\{H_{\omega}\right\}_{\omega \in \Omega}$ is a family of Hilbert spaces.

$\triangleleft$ We have

$$
A=A_{1} \oplus A_{2} \oplus \cdots \oplus A_{I_{\infty}} \oplus A_{I I_{1}} \oplus A_{I I_{\infty}} \oplus A_{I I I}
$$

and the subalgebra (without the part $A_{2}$ )

$$
A_{1} \oplus A_{3} \oplus A_{4} \oplus \cdots \oplus A_{I_{\infty}} \oplus A_{I I_{1}} \oplus A_{I I_{\infty}} \oplus A_{I I I}
$$

is reversible. The last statement can be proven similar to [9, Theorem 5.3.10]. By [10] the subalgebra $A_{2}$ can be represented as follows

$$
A_{2}=\sum_{i \in \Xi} C\left(X_{i}, \mathbf{R} \oplus H_{i}\right),
$$

where $\Xi$ is a set of indices, $\left\{X_{i}\right\}_{i \in \Xi}$ is a family of extremal compacts and $\left\{H_{i}\right\}_{i \in \Xi}$ is a family of Hilbert spaces. Hence by [9, Theorem 6.2.5] there exist central projections $h, g$ such that $A=h A \oplus g A, h A$ is reversible and $g A$ is totally nonreversible. For all $a, b_{1}, \ldots, b_{n}, c_{1}, \ldots, c_{m}$ in $h A$ we have

$$
b_{1} \ldots b_{n} a c_{1} \ldots c_{m}+c_{m} c_{m-1} \ldots c_{1} a b_{n} b_{n-1} \ldots b_{1} \in h A
$$


since $h A$ is reversible. Similarly for all $b, c \in R^{*}(h A), a \in h A$ we have

$$
b a c+c^{*} a b^{*} \in h A .
$$

Hence $h A=J$.

By Proposition 3.5 there exist two central projections $e, f$ in $h A$ and a norm-closed two sided ideal $I$ of $C^{*}(h A)$ such that $e+f=h, e A={ }^{\perp}\left({ }^{\perp}\left(I_{s a}\right)_{+}\right)_{+}, f A$ is a reversible AJWalgebra and $R^{*}(f A) \cap i R^{*}(f A)=\{0\}$. This completes the proof. $\triangleright$

Let $A$ be a special AJW-algebra. Despite the fact that for the real $\mathrm{AW}^{*}$-algebra $R^{*}(A)$ the $\mathrm{C}^{*}$-algebra $\mathscr{M}=R^{*}(A)+i R^{*}(A)$ is not necessarily a complex $\mathrm{AW}^{*}$-algebra we consider, that

Conjecture. Under the conditions of Theorem 3.8 the following equality is valid

$$
e A=I_{s a} .
$$

\section{References}

1. Albeverio S., Ayupov Sh. A. and Abduvaitov A. H. On Real AW*-algebras. Methods of Functional Analysis and Topology.-2005.-Vol. 11, № 2.-P. 99-112.

2. Albeverio S., Ayupov Sh. A. and Abduvaitov A. H. On the coincidence of types of a real AW*-algebra and its comlexification // News of Russian Acad. of Sci. Math. ser.-2004.-Vol. 68.-P. 3-12.-[Russian].

3. Arzikulov F. N. On abstract JW-algebras // Sib. Math. J.-1998.-Vol. 39.-P. 20-27.

4. Arzikulov F. N. On an analog of the Peirce decomposition // Sib. Math. J.-1999.-Vol. 40.-P. 413-419.

5. Arzikulov F. N. AJW-algebras of type I and their classification // Sib. Adv. Math.-1998.-Vol. 8.P. $30-48$.

6. Ayupov Sh. A. Traces on JW-algebras and enveloping $W^{*}$-algebras // Math. Z.-1987.-Vol. 194.P. $15-23$.

7. Ayupov Sh. Classification and representation of ordered Jordan algebras Fan.-Tashkent, 1986.[Russian].

8. Chilin V. I. Partial ordered Baer involutive algebras // Sovrem. Problemy Matematiki, Itogi nauki i tehkniki. VINITI.-1985.-Vol. 27.-P. 99-128.-[Russian].

9. Hanche-Olcen H., Størmer E. Jordan Operator Algebras.-Boston etc: Pitman Publ. Inc., 1984.

10. Kusraev A. G. On the structure of AJW-algebras of type $I_{2} / /$ Sib. Math. J.-1999.-Vol. 40.-P. 905917.

11. Li B. Real operator algebras. Institute of Mathematics.-China: Acad. Sinica Beijing, 2002.

12. Sakai S. $C^{*}$-algebras and $W^{*}$-algebras.-Springer, 1971.

13. Størmer E. Jordan algebras of type I // Acta. Math.-1966.-Vol. 115.-P. 165-184.

14. Topping D. M. Jordan algebras of self-adjoint operators // Mem. Am. Math. Soc.-1965.-Vol. 53.

Received September 24, 2015.

Ayupov Shavkat Abdullayevich

National University of Uzbekistan,

Director of the Institute of Math.

Do'rmon yo'li st., Tashkent, 1000125, Uzbekistan

Email: sh_ayupov@mail.ru

Arzikulov Farhodjon Nematjonovich

Andizhan State University,

Department of Mathematics docent,

University street, Andizhan, 710020, Uzbekistan

Email: arzikulovfn@rambler.ru 
ОБРАТИМЫЕ АЈW-АЛГЕБРЫ

Аюпов Ш. А., Арзикулов Ф. Н.

Основной результат статьи гласит, что каждая специальная AJW-алгебра раскладывается в прямую сумму тотально необратимой и обратимой подалгебр. В свою очередь, каждая обратимая AJWалгебра раскладывается в прямую сумму подалгебры, которая содержит идеал такой, что аннулятор комплексификации этого идеала в обертывающей $C^{*}$-алгебре этой подалгебры равен нулю и подалгебры, обертывающая вещественная алгебра фон Неймана которой является чисто вещественной.

Ключевые слова: AJW-алгебра, обратимая AJW-алгебра, $A W^{*}$-алгебра, обертывающая *-алгебра. 